\title{
Some new fuzzy fixed point theorems via distance functions with applications
}

\author{
Bitao Cheng ${ }^{a, b, c}$, Jianhua Chen $^{\mathrm{b}, *}$, Xianhua Tang $^{\mathrm{b}}$ \\ a School of Mathematics and Statistics, Qujing Normal University, Qujing, Yunnan, 655011, P. R. China. \\ ${ }^{b}$ School of Mathematics and Statistics, Central South University, Changsha, Hunan, 410083, P. R. China. \\ ${ }^{c}$ Institute of Applied Mathematics, Qujing Normal University, Qujing, Yunnan, 655011, P. R. China.
}

Communicated by Y. J. Cho

\begin{abstract}
In this paper, we prove some new fuzzy fixed point theorems on a space of fuzzy sets under a G-distance function and a $\mathrm{G}^{\prime}$-distance function. Our results extend, generalize, and improve some existing results. Moreover, some applications are given here to illustrate the usability of the obtained results. (C)2017 All rights reserved.
\end{abstract}

Keywords: Fuzzy set, fuzzy-mapping, G-distance functions, G'-distance functions, fuzzy fixed point. 2010 MSC: MSC 47H10, 54H25.

\section{Introduction and preliminaries}

It is well-known that functional analysis is made up of two main methods which are variational methods and fixed point methods. Variational methods are used to prove the existence of solutions for differential equations [10, 14, 15, 23, 32, 33, 35-37]. However, fixed point methods are studied by many scholars $[12,13]$ in different spaces. Recently, fuzzy fixed point has attracted wide attention. As far as we know, fuzzy set theory plays an important role in many scientific and engineering applications. The fuzziness appears when we need to perform, on manifold, calculations with imprecision variables. The concept of fuzzy sets was introduced initially by Zadeh [34] in 1965. Since then, Heilpern [21] defined the fuzzy mapping $T: X \rightarrow W_{\alpha}(X)$ and proved a fixed point theorem for fuzzy mapping $T$ in metric linear space, which is a fuzzy extension of the Banach contraction principle. Subsequently, several other authors $[1-9,11,16,17,20-22,24-26,29,30,34]$ have studied existence of fixed points of fuzzy mappings satisfying some different contractive type conditions.

On the one hand, in 2008, Qiu et al. [27] defined the fuzzy mapping $F: \mathcal{C B}(X) \rightarrow \mathcal{C B}(X)$ on a space of fuzzy sets and proved a fixed point theorem for fuzzy mappings $F$ in complete metric spaces and this is different from the approach which is used by Heilpern [21]. On the other hand, in 2009, Qiu et al. [28]

\footnotetext{
*Corresponding author

Email addresses: chengbitao@126.com (Bitao Cheng), cjh19881129@163.com (Jianhua Chen), tangxh@mail.csu.edu.cn (Xianhua Tang)

doi:10.22436/jnsa.010.04.56
} 
defined the fuzzy mapping $\mathrm{K}: \mathcal{C}(X) \rightarrow \mathcal{C}(X)$ (please see [28]) on a space of fuzzy sets in another way and proved a fixed point theorem for fuzzy mappings $\mathrm{K}$ in compact metric spaces. Recently, following Qiu's work, Suantai et al. [31] provided some fuzzy fixed point theorems on a space of fuzzy sets equipped with supremum metric by using $\Re$-functions.

Motivated by [11] and [31], in this paper, we prove some new fuzzy fixed point theorems on a space of fuzzy sets under a G-distance function and $\mathrm{G}^{\prime}$-distance function in complete and compact metric spaces. Our results extend, generalize and improve the results of [27, 28, 31]. Moreover, some applications are given here to illustrate the usability of the obtained results.

Throughout this paper, we shall use the following notions.

Let $(X, d)$ be a metric space, and let $\mathrm{CB}(X)$ be the set of all nonempty bounded closed subsets of $X$. Recall that the Hausdorff metric is a function $\mathrm{H}$ on $\mathrm{CB}(\mathrm{X})$ defined by

$$
H(A, B)=\max \left\{\sup _{x \in B} d(x, A), \sup _{x \in A} d(x, B)\right\}=\max \{\rho(B, A), \rho(A, B)\} \quad \text { for all } A, B \in C B(X),
$$

where $\rho(A, B)=\sup _{x \in A} d(x, B)$ is the Hausdorff separation of $A$ from $B$.

A fuzzy set $\mu$ in $X$ is a function with domain $X$ and values in $I=[0,1]$. If $\mu$ is a fuzzy set and $x \in X$, then the function value $\mu(x)$ is called the grade of membership of $x$ in $X$. The $\alpha$-cut set of $\mu$, denoted by $[\mu]_{\alpha}$, is defined as

$$
[\mu]_{\alpha}=\{x: \mu(x) \geqslant \alpha\},
$$

where $\alpha \in(0,1]$, and we separately specify the support $[\mu]_{0}$ of $\mu$ to be the closure of the union of $[\mu]_{\alpha}$ for $0<\alpha \leqslant 1$. We denote by $\mathcal{C} \mathcal{B}(X)$ the totality of fuzzy sets $\mu: X \rightarrow I$ for which, for each $\alpha \in I$, the $\alpha$-cut of $\mu$ is a nonempty closed bounded subset of $X$.

Let $\mu_{1}, \mu_{2} \in \mathcal{C B}(X)$. Then $\mu_{1}$ is said to be included in $\mu_{2}$, denoted by $\mu_{1} \subseteq \mu_{2}$, if and only if $\mu_{1}(x) \leqslant$ $\mu_{2}(x)$ for each $x \in X$. Thus we have $\mu_{1} \subseteq \mu_{2}$ if and only if $\left[\mu_{1}\right]_{\alpha} \subseteq\left[\mu_{2}\right]_{\alpha}$ for all $\alpha \in$ I. Let $X, Y$ be any metric space. A mapping $F$ is said to be a fuzzy mapping if and only if $F$ is a mapping from the space $\mathcal{C B}(X)$ into $\mathcal{C} \mathcal{B}(X)$, i.e., $F(\mu) \in \mathcal{C} \mathcal{B}(X)$ for each $\mu \in \mathcal{C} \mathcal{B}(X) . \mu_{*} \in \mathcal{C} \mathcal{B}(X)$ is said to be a fixed point of a fuzzy self-mapping $\mathrm{F}$ of $\mathcal{C B}(\mathrm{X})$ if and only if $\mu_{*} \subseteq \mathrm{F}\left(\mu_{*}\right)$.

The $\mathrm{d}_{\infty}$-metric (called supremum or generalized Hausdorff metric) is a metric on $\mathcal{C B}(X)$ which is defined as follows:

$$
\mathrm{d}_{\infty}\left(\mu_{1}, \mu_{2}\right)=\sup _{0 \leqslant \alpha \leqslant 1} \mathrm{H}\left(\left[\mu_{1}\right]_{\alpha},\left[\mu_{2}\right]_{\alpha}\right)=\max \left\{\rho_{\infty}\left(\mu_{1}, \mu_{2}\right), \rho_{\infty}\left(\mu_{2}, \mu_{1}\right)\right\},
$$

where $\mu_{1}, \mu_{2} \in \mathcal{C} \mathcal{B}(X)$, and

$$
\rho_{\infty}\left(\mu_{1}, \mu_{2}\right)=\sup _{0 \leqslant \alpha \leqslant 1} \rho\left(\left[\mu_{1}\right]_{\alpha},\left[\mu_{2}\right]_{\alpha}\right)
$$

is the Hausdorff separation of $\mu_{1}$ from $\mu_{2}$. Notice that the supremum in (1.1) may be not attained, and so it cannot be replaced by a maximum. To clarify this, we include the following example, which can be found in [18].

Example 1.1. Let $X$ be a set of real numbers and $\mu, v \in \mathcal{C B}(X)$ be fuzzy subsets of $X$ such that the corresponding level sets are

$$
[\mu]_{\alpha}=[v]_{\alpha}=[0,1] \text { for } 0 \leqslant \alpha \leqslant \frac{1}{2}
$$

and

$$
[\mu]_{\alpha}=\{0\},[v]_{\alpha}=[0,2(1-\alpha)] \text { for } \frac{1}{2} \leqslant \alpha \leqslant 1 .
$$

It follows that

$$
H\left([\mu]_{\alpha},[v]_{\alpha}\right)= \begin{cases}0, & \text { for } 0 \leqslant \alpha \leqslant \frac{1}{2}, \\ 2(1-\alpha), & \text { for } \frac{1}{2} \leqslant \alpha \leqslant 1 .\end{cases}
$$

Hence, $d_{\infty}(\mu, v)=\sup _{0 \leqslant \alpha \leqslant 1} H\left([\mu]_{\alpha},[v]_{\alpha}\right)=1$, but this is not attained. 
Note that if $\left\{\mu_{n}\right\}$ is a sequence in $\mathcal{C B}(X)$, then it follows from the definition of $d_{\infty}$ that $\left\{\mu_{n}\right\}$ converges with respect to the $d_{\infty}$-metric if and only if $\left[\mu_{n}\right]_{\alpha}$ converges uniformly in $\alpha \in I$ with respect to the Hausdorff metric. Further, we know that the metric space $\left(\mathcal{C B}(X), \mathrm{d}_{\infty}\right)$ and $\left(\mathcal{C}(X), \mathrm{d}_{\infty}\right)$ are complete provided $(X, d)$ is complete (see Theorem 1 in [27] and Theorem 1 in [28]). Now, we list some results of the $d_{\infty}$-metric as follows.

Lemma $1.2([27,28])$. Let $\mu_{1}, \mu_{2}, \mu_{3} \in \mathcal{C B}(X)$ (or $\mathcal{C}(X)$ ). Then the following properties hold:

(i) $\rho\left(\mu_{1}, \mu_{2}\right)=0$ if and only if $\mu_{1} \subseteq \mu_{2}$,

(ii) if $\mu_{1} \subseteq \mu_{2}$, then $\rho_{\infty}\left(\mu_{1}, \mu_{3}\right) \leqslant d_{\infty}\left(\mu_{2}, \mu_{3}\right)$,

(iii) $\rho_{\infty}\left(\mu_{1}, \mu_{3}\right) \leqslant d_{\infty}\left(\mu_{1}, \mu_{2}\right)+\rho_{\infty}\left(\mu_{2}, \mu_{3}\right)$.

Lemma 1.3 ([27]). Let $(X, d)$ be a metric space and $\mu_{1}, \mu_{2} \in \mathcal{C B}(X)$. Then for any $\beta>1$ and any $\mu_{3} \in \mathcal{C} \mathcal{B}(X)$ satisfying $\mu_{3} \subseteq \mu_{1}$, there exists a $\mu_{4} \in \mathcal{C B}(X)$ such that $\mu_{4} \subseteq \mu_{2}$ and $\mathrm{d}_{\infty}\left(\mu_{3}, \mu_{4}\right) \leqslant \beta \mathrm{d}_{\infty}\left(\mu_{1}, \mu_{2}\right)$.

Lemma 1.4 ([28]). Let $(X, d)$ be a metric space and $\mu_{1}, \mu_{2} \in \mathfrak{C}(X)$. Then for any $\mu_{3} \in \mathfrak{C}(X)$ satisfying $\mu_{3} \subseteq \mu_{1}$, there exists a $\mu_{4} \in \mathfrak{C}(X)$ such that $\mu_{4} \subseteq \mu_{2}$ and $\mathrm{d}_{\infty}\left(\mu_{3}, \mu_{4}\right) \leqslant \mathrm{d}_{\infty}\left(\mu_{1}, \mu_{2}\right)$.

In [31], the authors gave an important tool related to our considered class of mappings. A function $\varphi:[0, \infty) \rightarrow[0,1)$ is said to be an $\mathfrak{R}$-function if

$$
\sup _{s \rightarrow t^{+}} \varphi(s)<1 \quad \text { for all } t \in[0, \infty) .
$$

Note that if $\varphi:[0, \infty) \rightarrow[0,1)$ is a non-decreasing function or a non-increasing function, then $\varphi$ is an $\mathfrak{R}$-function. This means the set of $\mathfrak{R}$-functions is a rich class. In [19], Du proved some of the following properties for the class of $\mathfrak{R}$-functions.

Theorem 1.5. Let $\varphi:[0, \infty) \rightarrow[0,1)$ be a function. Then the following statements are equivalent

(a) $\varphi$ is an $\mathfrak{R}$-function.

(b) For any nonincreasing sequence $\left\{x_{n}\right\}_{n \in N}$ in $[0, \infty)$, we have $0 \leqslant \sup _{n \in N} \varphi\left(x_{n}\right)<1$.

By Lemma 1.2, Lemma 1.3, and Theorem 1.5, Qiu et al. [27] and Suantai et al. [31] proved the following common fixed point theorems under the assumption of a closed bounded cut set of $\mathcal{C B}(X)$.

Theorem 1.6. Let $(\mathrm{X}, \mathrm{d})$ be a complete metric space and let $\left\{\mathrm{F}_{\mathrm{i}}\right\}_{i=1}^{\infty}$ be a sequence of self-mappings of $\mathcal{C B}(\mathrm{X})$. If there exists a constant $q \in(0,1)$ such that for each $\mu_{1}, \mu_{2} \in \mathcal{C B}(X)$, and for arbitrary positive integers $i$ and $j$, $i \neq j$,

$$
d_{\infty}\left(F_{i}\left(\mu_{1}\right), F_{j}\left(\mu_{2}\right)\right) \leqslant q M_{i, j}\left(\mu_{1}, \mu_{2}\right),
$$

where

$$
M_{i, j}\left(\mu_{1}, \mu_{2}\right)=\max \left\{d_{\infty}\left(\mu_{1}, \mu_{2}\right), \rho_{\infty}\left(\mu_{1}, F_{i}\left(\mu_{1}\right)\right), \rho_{\infty}\left(\mu_{2}, F_{j}\left(\mu_{2}\right)\right), \frac{\rho_{\infty}\left(\mu_{2}, F_{i}\left(\mu_{1}\right)\right)+\rho_{\infty}\left(\mu_{1}, F_{j}\left(\mu_{2}\right)\right)}{2}\right\},
$$

then there exists a $\mu_{*} \in \mathcal{C B}(X)$ such that $\mu_{*} \subseteq \mathrm{F}_{\mathfrak{i}}\left(\mu_{*}\right)$ for all $i \in N$.

Theorem 1.7. Let $(\mathrm{X}, \mathrm{d})$ be a complete metric space and let $\left\{\mathrm{F}_{i}\right\}_{i=1}^{\infty}$ be a sequence of self-mappings of $\mathrm{CB}(\mathrm{X})$. Assume that there exists an $\mathfrak{R}$-function $\varphi:[0, \infty) \rightarrow[0,1)$ such that for each $\mu_{1}, \mu_{2} \in \mathcal{C B}(X)$, and for arbitrary positive integers $i$ and $j, i \neq j$,

$$
d_{\infty}\left(F_{i}\left(\mu_{1}\right), F_{j}\left(\mu_{2}\right)\right) \leqslant \varphi\left(d_{\infty}\left(\mu_{1}, \mu_{2}\right)\right) M_{i, j}\left(\mu_{1}, \mu_{2}\right),
$$

where

$$
M_{i, j}\left(\mu_{1}, \mu_{2}\right)=\max \left\{d_{\infty}\left(\mu_{1}, \mu_{2}\right), \rho_{\infty}\left(\mu_{1}, F_{i}\left(\mu_{1}\right)\right), \rho_{\infty}\left(\mu_{2}, F_{j}\left(\mu_{2}\right)\right), \frac{\rho_{\infty}\left(\mu_{2}, F_{i}\left(\mu_{1}\right)\right)+\rho_{\infty}\left(\mu_{1}, F_{j}\left(\mu_{2}\right)\right)}{2}\right\} .
$$

Then there exists a $\mu_{*} \in \mathcal{C B}(X)$ such that $\mu_{*} \subseteq \mathrm{F}_{i}\left(\mu_{*}\right)$ for all $i \in N$. 
By Lemmas 1.2 and 1.3, Qiu et al. [28] proved the following common fixed point theorems under the assumption of a compact cut set of $\mathfrak{C}(\mathrm{X})$.

Theorem 1.8. Let $(\mathrm{X}, \mathrm{d})$ be a compact metric space and let $\left\{\mathrm{F}_{i}\right\}_{i=1}^{\infty}$ be a sequence of self-mappings of $\mathcal{C}(\mathrm{X})$. Let $\Phi:[0, \infty) \rightarrow[0, \infty)$ be a non-decreasing function satisfying the following condition: $\Phi$ is continuous from the right and

$$
\sum_{\mathrm{n}=1}^{\infty} \Phi^{\mathrm{n}}(\mathrm{t})<\infty \text { for all } \mathrm{t}>0,
$$

where $\Phi^{n}$ denotes the $n$th iterative function of $\Phi$. Suppose that for arbitrary positive integers $i$ and $j, i \neq j$,

$$
\mathrm{d}_{\infty}\left(\mathrm{F}_{\mathfrak{i}}\left(\mu_{1}\right), \mathrm{F}_{\mathbf{j}}\left(\mu_{2}\right)\right) \leqslant \Phi\left(M_{i, j}\left(\mu_{1}, \mu_{2}\right)\right),
$$

where

$$
M_{i, j}\left(\mu_{1}, \mu_{2}\right)=\max \left\{d_{\infty}\left(\mu_{1}, \mu_{2}\right), \rho_{\infty}\left(\mu_{1}, F_{i}\left(\mu_{1}\right)\right), \rho_{\infty}\left(\mu_{2}, F_{j}\left(\mu_{2}\right)\right), \frac{\rho_{\infty}\left(\mu_{2}, F_{i}\left(\mu_{1}\right)\right)+\rho_{\infty}\left(\mu_{1}, F_{j}\left(\mu_{2}\right)\right)}{2}\right\} .
$$

Then there exists a $\mu_{*} \in \mathcal{E B}(X)$ such that $\mu_{*} \subseteq \mathrm{F}_{i}\left(\mu_{*}\right)$ for all $i \in N$.

Next, we introduce some classes of functions.

Let $\Psi$ be the set of all functions $\phi$ such that $\phi:[0,+\infty) \rightarrow[0,+\infty)$ be a continuous and nondecreasing function with $\phi(t)=0$ if and only if $t=0$.

Let $\checkmark$ be the set of all functions $\eta$ such that $\eta:[0,+\infty) \rightarrow[0,+\infty)$ be lower semi continuous with $\eta(t)=0$ if and only if $t=0$.

Let $\Omega$ be the set of all functions $\psi$ such that $\psi:[0,+\infty) \rightarrow[0,+\infty)$ be nondecreasing and continuous from the right with $\lim _{n \rightarrow \infty} \psi^{n}(t)=0$ for all $t \in(0,+\infty)$. If $\psi \in \Omega$, then $\psi$ is called $\Omega$-map, then it is an easy matter to show that

(1) $\psi(t)<t$ for all $t \in(0,+\infty)$,

(2) $\psi(0)=0$.

Remark 1.9. By Theorem 1.8 and the above function classes, it is an easy matter to show that $\Phi \in \Omega$.

\section{Fuzzy fixed point theorems under a G-distance function}

In this section, inspired by Constantin [17] and Chen et al. [11], we will show some fuzzy fixed point theorems on a space of fuzzy sets via a G-distance function. In what follows, we slightly modified the definition of G-distance functions which was introduced by Chen et al. [11].

Definition 2.1. A function $g$ is said to be a G-distance function if $g:[0, \infty)^{5} \rightarrow[0, \infty)$ is continuous function with the following properties hold:

(i) $g$ is nondecreasing in the $2^{\text {nd }}, 3^{\text {rd }}, 4^{\text {th }}$, and $5^{\text {th }}$ variables;

(ii) if $u, v \in[0, \infty)$ are such that $u \leqslant g(v, v, u, 0, u+v)$ or $u \leqslant g(v, u, v, 0, u+v)$ then $u \leqslant h v$ where $0<\mathrm{h}<1$ is a given constant;

(iii) if $u \in[0, \infty)$ is such that $u \leqslant g(u, 0,0, u, u)$, then $u=0$.

Next, we introduce and prove the following result which generalizes some existing results.

Theorem 2.2. Let $(\mathrm{X}, \mathrm{d})$ be a complete metric space and $\mathrm{g}$ be a $\mathrm{G}$-distance function and $\left\{\mathrm{F}_{i}\right\}_{i=1}^{\infty}$ a sequence of selfmappings of $\mathcal{C B}(X)$. Suppose that there exists an $\mathfrak{R}$-function $\varphi:[0, \infty) \rightarrow[0,1)$ such that for each $\mu_{1}, \mu_{2} \in \mathcal{C} \mathcal{B}(X)$, and for arbitrary positive integers $i$ and $j, i \neq j$,

$$
d_{\infty}\left(F_{i}\left(\mu_{1}\right), F_{j}\left(\mu_{2}\right)\right) \leqslant \varphi\left(d_{\infty}\left(\mu_{1}, \mu_{2}\right)\right) M\left(\mu_{1}, \mu_{2}\right),
$$

where

$$
M\left(\mu_{1}, \mu_{2}\right)=g\left(d_{\infty}\left(\mu_{1}, \mu_{2}\right), \rho_{\infty}\left(\mu_{1}, F_{i}\left(\mu_{1}\right)\right), \rho_{\infty}\left(\mu_{2}, F_{j}\left(\mu_{2}\right)\right), \rho_{\infty}\left(\mu_{2}, F_{i}\left(\mu_{1}\right)\right), \rho_{\infty}\left(\mu_{1}, F_{j}\left(\mu_{2}\right)\right)\right) .
$$

Then there exists a $\mu_{*} \in \mathcal{C B}(X)$ such that $\mu_{*} \subseteq \mathrm{F}_{i}\left(\mu_{*}\right)$ for all $i \in N$. 
Proof. Let $\mu_{0} \in \mathcal{C} \mathcal{B}(\mathrm{X})$, and $\mu_{1} \subseteq \mathrm{F}_{1}\left(\mu_{0}\right)$, by Lemma 1.2 (ii), we can get

$$
\rho_{\infty}\left(\mu_{0}, F_{1}\left(\mu_{0}\right)\right) \leqslant d_{\infty}\left(\mu_{0}, \mu_{1}\right) .
$$

By induction, we produce a sequence $\left\{\mu_{n}\right\}$ of points of $\mathcal{C B}(X)$ such that

$$
\left\{\begin{array}{l}
\mu_{n+1} \subseteq F_{n+1}\left(\mu_{n}\right), \\
\rho_{\infty}\left(\mu_{n}, F_{n+1}\left(\mu_{n}\right)\right) \leqslant d_{\infty}\left(\mu_{n}, \mu_{n+1}\right) .
\end{array}\right.
$$

Let us define a function $k:[0, \infty) \rightarrow[0,1)$ by

$$
k(t)=\frac{1+\varphi(t)}{2} \text { for all } t \in[0, \infty) .
$$

Note that we have $0 \leqslant \varphi(t)<k(t)<1$ for all $t \in[0, \infty)$.

We will start by picking a fuzzy set $\mu_{0} \in \mathcal{C B}(X)$. We subsequently choose $\mu_{1} \subseteq \mathrm{F}_{1}\left(\mu_{0}\right)$ and a positive real number $\epsilon_{0}$ such that $\epsilon_{0} \in\left(\frac{1-k\left(d_{\infty}\left(\mu_{0}, \mu_{1}\right)\right)}{2}, 1-k\left(d_{\infty}\left(\mu_{0}, \mu_{1}\right)\right)\right)$. Next, by using this $\epsilon_{0}$, we can find a positive real number $\beta_{0}$ such that $\beta_{0} \in\left(1, \frac{1-\epsilon_{0}}{k\left(\mathrm{~d}_{\infty}\left(\mu_{0}, \mu_{1}\right)\right)}\right)$. Now, by Lemma 1.3, there exists $\mu_{2} \in \mathcal{C B}(X)$ such that $\mu_{2} \subseteq \mathrm{F}_{2}\left(\mu_{1}\right)$ and

$$
d_{\infty}\left(\mu_{1}, \mu_{2}\right) \leqslant \beta_{0} d_{\infty}\left(F_{1}\left(\mu_{0}\right), F_{2}\left(\mu_{1}\right)\right) .
$$

Now, We choose $\mu_{2} \subseteq F_{2}\left(\mu_{1}\right)$ and a positive real number $\epsilon_{1}$ such that $\epsilon_{1} \in\left(\frac{1-k\left(d_{\infty}\left(\mu_{1}, \mu_{2}\right)\right)}{2}, 1-\right.$ $\left.k\left(d_{\infty}\left(\mu_{1}, \mu_{2}\right)\right)\right)$. Next, by using this $\epsilon_{1}$, we can find a positive real number $\beta_{1}$ such that $\beta_{1} \in\left(1, \frac{1-\epsilon_{1}}{k\left(d_{\infty}\left(\mu_{1}, \mu_{2}\right)\right)}\right)$. Now, by Lemma 1.3 , there exists $\mu_{3} \in \mathcal{C B}(X)$ such that $\mu_{3} \subseteq F_{3}\left(\mu_{2}\right)$ and

$$
d_{\infty}\left(\mu_{2}, \mu_{3}\right) \leqslant \beta_{1} d_{\infty}\left(F_{2}\left(\mu_{1}\right), F_{3}\left(\mu_{2}\right)\right) .
$$

By induction, we produce two sequences of points of $\left\{\epsilon_{n}\right\}$ and $\left\{\beta_{n}\right\}$ and a sequence $\left\{\mu_{n}\right\}$ in $\mathcal{C B}(X)$ such that

$$
\left\{\begin{array}{l}
\mu_{n+1} \subseteq F_{n+1}\left(\mu_{n}\right), \\
d_{\infty}\left(\mu_{n+1}, \mu_{n+2}\right) \leqslant \beta_{n} d_{\infty}\left(F_{n+1}\left(\mu_{n}\right), F_{n+2}\left(\mu_{n+1}\right)\right) \\
\epsilon_{n} \in\left(\frac{1-k\left(d_{\infty}\left(\mu_{n}, \mu_{n+1}\right)\right)}{2}, 1-k\left(d_{\infty}\left(\mu_{n}, \mu_{n+1}\right)\right)\right) \\
\beta_{n} \in\left(1, \frac{1-\epsilon_{n}}{k\left(d_{\infty}\left(\mu_{n}, \mu_{n+1}\right)\right)}\right)
\end{array}\right.
$$

for all $n \in \mathbf{N}$.

Next, we prove that $\left\{\mu_{n}\right\}$ is a Cauchy sequence in $\mathcal{C B}(X)$. In fact, for arbitrary positive integer $n$, by inequalities (2.1), (2.2), and the formula (2.3), we have

$$
\begin{aligned}
d_{\infty}\left(\mu_{n+1}, \mu_{n+2}\right) & \leqslant \beta_{n} d_{\infty}\left(F_{n+1}\left(\mu_{n}\right), F_{n+2}\left(\mu_{n+1}\right)\right) \\
& \leqslant \beta_{n} \varphi\left(d_{\infty}\left(\mu_{n}, \mu_{n+1}\right)\right) M\left(\mu_{n}, \mu_{n+1}\right) \\
& <\beta_{n} k\left(d_{\infty}\left(\mu_{n}, \mu_{n+1}\right)\right) M\left(\mu_{n}, \mu_{n+1}\right),
\end{aligned}
$$

where

$$
\begin{aligned}
M\left(\mu_{n}, \mu_{n+1}\right)= & g\left(d_{\infty}\left(\mu_{n}, \mu_{n+1}\right), \rho_{\infty}\left(\mu_{n}, F_{n+1}\left(\mu_{n}\right)\right)\right. \\
& \left., \rho_{\infty}\left(\mu_{n+1}, F_{n+2}\left(\mu_{n+1}\right)\right), \rho_{\infty}\left(\mu_{n+1}, F_{n+1}\left(\mu_{n}\right)\right), \rho_{\infty}\left(\mu_{n}, F_{n+2}\left(\mu_{n+1}\right)\right)\right) \\
\leqslant & g\left(d_{\infty}\left(\mu_{n}, \mu_{n+1}\right), d_{\infty}\left(\mu_{n}, \mu_{n+1}\right), d_{\infty}\left(\mu_{n+1}, \mu_{n+2}\right)\right. \\
& \left., d_{\infty}\left(\mu_{n+1}, \mu_{n+1}\right), \rho_{\infty}\left(\mu_{n}, F_{n+2}\left(\mu_{n+1}\right)\right)\right) \\
\leqslant & g\left(d_{\infty}\left(\mu_{n}, \mu_{n+1}\right), d_{\infty}\left(\mu_{n}, \mu_{n+1}\right), d_{\infty}\left(\mu_{n+1}, \mu_{n+2}\right), 0, \rho_{\infty}\left(\mu_{n}, F_{n+2}\left(\mu_{n+1}\right)\right)\right) \\
\leqslant & g\left(d_{\infty}\left(\mu_{n}, \mu_{n+1}\right), d_{\infty}\left(\mu_{n}, \mu_{n+1}\right), d_{\infty}\left(\mu_{n+1}, \mu_{n+2}\right), 0\right. \\
& \left., d_{\infty}\left(\mu_{n}, \mu_{n+1}\right)+\rho_{\infty}\left(\mu_{n+1}, F_{n+2}\left(\mu_{n+1}\right)\right)\right) \\
\leqslant & g\left(d_{\infty}\left(\mu_{n}, \mu_{n+1}\right), d_{\infty}\left(\mu_{n}, \mu_{n+1}\right), d_{\infty}\left(\mu_{n+1}, \mu_{n+2}\right), 0\right. \\
& \left., d_{\infty}\left(\mu_{n}, \mu_{n+1}\right)+d_{\infty}\left(\mu_{n+1}, \mu_{n+2}\right)\right) .
\end{aligned}
$$


Since $0<\beta_{n} k\left(d_{\infty}\left(\mu_{n}, \mu_{n+1}\right)\right)<1-\epsilon_{n}<1$ for all $n \in \mathbf{N}$, by (2.4) and (2.5), then for all $n$, we have

$$
d_{\infty}\left(\mu_{n+1}, \mu_{n+2}\right)<g\left(d_{\infty}\left(\mu_{n}, \mu_{n+1}\right), d_{\infty}\left(\mu_{n}, \mu_{n+1}\right), d_{\infty}\left(\mu_{n+1}, \mu_{n+2}\right), 0, d_{\infty}\left(\mu_{n}, \mu_{n+1}\right)+d_{\infty}\left(\mu_{n+1}, \mu_{n+2}\right)\right)
$$

From Definition 2.1 (ii), we can conclude that

$$
d_{\infty}\left(\mu_{n+1}, \mu_{n+2}\right) \leqslant h d_{\infty}\left(\mu_{n}, \mu_{n+1}\right),
$$

where $0<\mathrm{h}<1$. By iteration, we have

$$
d_{\infty}\left(\mu_{n}, \mu_{n+1}\right) \leqslant h d\left(x_{n-1}, x_{n}\right) \leqslant \cdots \leqslant h^{n} d\left(\mu_{0}, \mu_{1}\right) .
$$

Furthermore, for $m>n$,

$$
\begin{aligned}
d\left(\mu_{n}, \mu_{m}\right) & \leqslant d_{\infty}\left(\mu_{n}, \mu_{n+1}\right)+d_{\infty}\left(\mu_{n+1}, \mu_{n+2}\right)+\cdots+d_{\infty}\left(\mu_{m-1}, \mu_{m}\right) \\
& \leqslant\left(h^{n}+h^{n-1}+\cdots+h^{m-1}\right) d_{\infty}\left(\mu_{0}, \mu_{1}\right) \leqslant \frac{h^{n}}{1-h} d_{\infty}\left(\mu_{0}, \mu_{1}\right) .
\end{aligned}
$$

It follows that $\left\{\mu_{n}\right\}$ is a Cauchy sequence in $\mathcal{C B}(X)$. Since $X$ is complete, it implies that $\left(\mathcal{C B}(X), d_{\infty}\right)$ is complete. Thus there exists a $\mu_{*}$ such that $\lim _{n \rightarrow \infty} \mu_{n}=x_{*}$. Next, we show that $\left\{\mu_{*}\right\} \subset F_{i} \mu_{*}$ for all $i \in \mathbf{N}$.

Let $i \in \mathbf{N}$ be arbitrary. By (ii) and (iii) in Lemma 1.2, we can get

$$
\rho_{\infty}\left(\mu_{*}, F_{i}\left(\mu_{*}\right)\right) \leqslant d_{\infty}\left(\mu_{*}, \mu_{j}\right)+\rho_{\infty}\left(\mu_{j}, F_{i}\left(\mu_{*}\right)\right) \leqslant d_{\infty}\left(\mu_{*}, \mu_{j}\right)+d_{\infty}\left(F_{j}\left(\mu_{j-1}\right), F_{i}\left(\mu_{*}\right)\right),
$$

since $\mu_{j} \in F_{j}\left(\mu_{j-1}\right)$ for arbitrary natural numbers $j$ such that $i \neq j$. Subsequently, by using (2.1), (2.6), and (iii) in Lemma 1.2, we have

$$
\begin{aligned}
d_{\infty}\left(F_{j}\left(\mu_{j-1}\right), F_{i}\left(\mu_{*}\right)\right) \leqslant & \varphi\left(d_{\infty}\left(\mu_{j-1}, \mu_{*}\right)\right) g\left(d_{\infty}\left(\mu_{j-1}, \mu_{*}\right), \rho_{\infty}\left(\mu_{j-1}, F_{j}\left(\mu_{j-1}\right)\right)\right. \\
& \left., \rho_{\infty}\left(\mu_{*}, F_{i}\left(\mu_{*}\right)\right), \rho_{\infty}\left(\mu_{*}, F_{j}\left(\mu_{j-1}\right)\right), \rho_{\infty}\left(\mu_{j-1}, F_{i}\left(\mu_{*}\right)\right)\right) \\
\leqslant & \varphi\left(d_{\infty}\left(\mu_{j-1}, \mu_{*}\right)\right) g\left(d_{\infty}\left(\mu_{j-1}, \mu_{*}\right), \rho_{\infty}\left(\mu_{j-1}, F_{j}\left(\mu_{j-1}\right)\right)\right. \\
& , d_{\infty}\left(\mu_{*}, \mu_{j}\right)+\rho_{\infty}\left(\mu_{j}, F_{i}\left(\mu_{*}\right)\right) \\
& \left., d_{\infty}\left(\mu_{*}, \mu_{j}\right)+\rho_{\infty}\left(\mu_{j}, F_{j}\left(\mu_{j-1}\right)\right), d_{\infty}\left(\mu_{j-1}, \mu_{*}\right)+\rho_{\infty}\left(\mu_{*}, F_{i}\left(\mu_{*}\right)\right)\right) \\
< & g\left(d_{\infty}\left(\mu_{j-1}, \mu_{*}\right), d_{\infty}\left(\mu_{j-1}, \mu_{j}\right), d_{\infty}\left(\mu_{*}, \mu_{j}\right)+\rho_{\infty}\left(\mu_{j}, F_{i}\left(\mu_{*}\right)\right)\right. \\
& \left., d_{\infty}\left(\mu_{*}, \mu_{j}\right)+d_{\infty}\left(\mu_{j}, \mu_{j}\right), d_{\infty}\left(\mu_{j-1}, \mu_{*}\right)+\rho_{\infty}\left(\mu_{*}, F_{i}\left(\mu_{*}\right)\right)\right) .
\end{aligned}
$$

Letting $j \rightarrow \infty$ in inequalities (2.6) and (2.7), we obtain

$$
\rho_{\infty}\left(\mu_{*}, F_{i}\left(\mu_{*}\right)\right)<g\left(0,0, \rho_{\infty}\left(\left(\mu_{*}\right), F_{i}\left(\mu_{*}\right)\right), 0, \rho_{\infty}\left(\mu_{*}, F_{i}\left(\mu_{*}\right)\right)\right) .
$$

Using (ii) in Definition 2.1, we can get $\rho_{\infty}\left(\mu_{*}, F_{i} \mu_{*}\right)=0$. Therefore, we have $\left\{\mu_{*}\right\} \subset F_{i} \mu_{*}$.

Remark 2.3.

(1) If we choose $\varphi(t)=\lambda(0<\lambda<1)$ and $g\left(x_{1}, x_{2}, x_{3}, x_{4}, x_{5}\right)=\delta \max \left\{x_{1}, x_{2}, x_{3}, \frac{x_{4}+x_{5}}{2}\right\}(0<\delta<1)$, then by Theorem 2.2, As long as we take $0<\mathrm{q}=\lambda \delta<1$, then we can get Theorem 1.6.

(2) Since we are considering a larger class of G-distance function, Theorem 2.2 improves Theorem 1.7.

Next, we want to give some results by using a G-distance function in compact metric spaces.

Theorem 2.4. Let $(\mathrm{X}, \mathrm{d})$ be a compact metric space and $\mathrm{g}$ be a $\mathrm{G}$-distance function and $\left\{\mathrm{F}_{\mathrm{i}}\right\}_{i=1}^{\infty}$ a sequence of selfmappings of $\mathcal{C}(X)$. Suppose that there exist $\phi \in \Psi$ and $\mathrm{L} \geqslant 0$ such that for each $\mu_{1}, \mu_{2} \in \mathcal{C}(X)$, and for arbitrary positive integers $i$ and $j, i \neq j$,

$$
\phi\left(d_{\infty}\left(F_{i}\left(\mu_{1}\right), F_{j}\left(\mu_{2}\right)\right)\right) \leqslant \phi\left(M\left(\mu_{1}, \mu_{2}\right)\right)+L N\left(\mu_{1}, \mu_{2}\right),
$$


where

$$
\begin{aligned}
M\left(\mu_{1}, \mu_{2}\right) & =g\left(d_{\infty}\left(\mu_{1}, \mu_{2}\right), \rho_{\infty}\left(\mu_{1}, F_{i}\left(\mu_{1}\right)\right), \rho_{\infty}\left(\mu_{2}, F_{j}\left(\mu_{2}\right)\right), \rho_{\infty}\left(\mu_{2}, F_{i}\left(\mu_{1}\right)\right), \rho_{\infty}\left(\mu_{1}, F_{j}\left(\mu_{2}\right)\right)\right), \\
N\left(\mu_{1}, \mu_{2}\right) & =\min \left\{d_{\infty}\left(\mu_{1}, \mu_{2}\right), \rho_{\infty}\left(\mu_{1}, F_{i}\left(\mu_{1}\right)\right), \rho_{\infty}\left(\mu_{2}, F_{j}\left(\mu_{2}\right)\right), \rho_{\infty}\left(\mu_{2}, F_{i}\left(\mu_{1}\right)\right), \rho_{\infty}\left(\mu_{1}, F_{j}\left(\mu_{2}\right)\right)\right\} .
\end{aligned}
$$

Then there exists a $\mu_{*} \in \mathcal{C}(X)$ such that $\mu_{*} \subseteq \mathrm{F}_{i}\left(\mu_{*}\right)$ for all $i \in N$.

Proof. Let $\mu_{0} \in \mathcal{C}(X)$, and $\mu_{1} \subseteq F_{1}\left(\mu_{0}\right)$, by Lemma 1.4, there exists a $\mu_{2}$ such that $\mu_{2} \subseteq F_{2}\left(\mu_{1}\right)$ and

$$
d_{\infty}\left(\mu_{1}, \mu_{2}\right) \leqslant d_{\infty}\left(F_{1}\left(\mu_{0}\right), F_{2}\left(\mu_{1}\right)\right) .
$$

Again by Lemma 1.4, we can find $\mu_{3} \in \mathcal{C}(X)$ such that $\mu_{3} \subseteq F_{3}\left(\mu_{2}\right)$,

$$
d_{\infty}\left(\mu_{2}, \mu_{3}\right) \leqslant d_{\infty}\left(F_{2}\left(\mu_{1}\right), F_{3}\left(\mu_{2}\right)\right) .
$$

By induction, we produce a sequence $\left\{\mu_{n}\right\}$ of points of $\mathcal{C}(X)$ such that

$$
\left\{\begin{array}{l}
\mu_{n+2} \subseteq F_{n+2}\left(\mu_{n+1}\right), \\
d_{\infty}\left(\mu_{n+1}, \mu_{n+2}\right) \leqslant d_{\infty}\left(F_{n+1}\left(\mu_{n}\right), F_{n+2}\left(\mu_{n+1}\right)\right) .
\end{array}\right.
$$

Next, we prove that $\left\{\mu_{n}\right\}$ is a Cauchy sequence in $\mathcal{C}(X)$. In fact, for arbitrary positive integer $n$, by inequality (2.8), formula (2.9), and the properties of $\phi$, we have

$$
\phi\left(d_{\infty}\left(\mu_{n+1}, \mu_{n+2}\right)\right) \leqslant \phi\left(d_{\infty}\left(F_{n+1}\left(\mu_{n}\right), F_{n+2}\left(\mu_{n+1}\right)\right)\right) \leqslant \phi\left(M\left(\mu_{n}, \mu_{n+1}\right)\right)+L N\left(\mu_{n}, \mu_{n+1}\right),
$$

where

$$
\begin{aligned}
M\left(\mu_{n}, \mu_{n+1}\right)= & g\left(d_{\infty}\left(\mu_{n}, \mu_{n+1}\right), \rho_{\infty}\left(\mu_{n}, F_{n+1}\left(\mu_{n}\right)\right)\right. \\
& \left., \rho_{\infty}\left(\mu_{n+1}, F_{n+2}\left(\mu_{n+1}\right)\right), \rho_{\infty}\left(\mu_{n+1}, F_{n+1}\left(\mu_{n}\right)\right), \rho_{\infty}\left(\mu_{n}, F_{n+2}\left(\mu_{n+1}\right)\right)\right) \\
\leqslant & g\left(d_{\infty}\left(\mu_{n}, \mu_{n+1}\right), d_{\infty}\left(\mu_{n}, \mu_{n+1}\right), d_{\infty}\left(\mu_{n+1}, \mu_{n+2}\right)\right. \\
& \left., d_{\infty}\left(\mu_{n+1}, \mu_{n+1}\right), \rho_{\infty}\left(\mu_{n}, F_{n+2}\left(\mu_{n+1}\right)\right)\right) \\
\leqslant & g\left(d_{\infty}\left(\mu_{n}, \mu_{n+1}\right), d_{\infty}\left(\mu_{n}, \mu_{n+1}\right), d_{\infty}\left(\mu_{n+1}, \mu_{n+2}\right), 0, \rho_{\infty}\left(\mu_{n}, F_{n+2}\left(\mu_{n+1}\right)\right)\right) \\
\leqslant & g\left(d_{\infty}\left(\mu_{n}, \mu_{n+1}\right), d_{\infty}\left(\mu_{n}, \mu_{n+1}\right), d_{\infty}\left(\mu_{n+1}, \mu_{n+2}\right), 0\right. \\
& \left., d_{\infty}\left(\mu_{n}, \mu_{n+1}\right)+\rho_{\infty}\left(\mu_{n+1}, F_{n+2}\left(\mu_{n+1}\right)\right)\right) \\
\leqslant & g\left(d_{\infty}\left(\mu_{n}, \mu_{n+1}\right), d_{\infty}\left(\mu_{n}, \mu_{n+1}\right), d_{\infty}\left(\mu_{n+1}, \mu_{n+2}\right), 0\right. \\
& \left., d_{\infty}\left(\mu_{n}, \mu_{n+1}\right)+d_{\infty}\left(\mu_{n+1}, \mu_{n+2}\right)\right) \\
N\left(\mu_{n}, \mu_{n+1}\right)= & \min \left\{d_{\infty}\left(\mu_{n}, \mu_{n+1}\right), \rho_{\infty}\left(\mu_{n}, F_{n+1}\left(\mu_{n}\right)\right)\right. \\
, & \left.\rho_{\infty}\left(\mu_{n+1}, F_{n+2}\left(\mu_{n+1}\right)\right), \rho_{\infty}\left(\mu_{n+1}, F_{n+1}\left(\mu_{n}\right)\right), \rho_{\infty}\left(\mu_{n}, F_{n+2}\left(\mu_{n+1}\right)\right)\right\} \\
\leqslant & \min \left\{d_{\infty}\left(\mu_{n}, \mu_{n+1}\right), d_{\infty}\left(\mu_{n}, \mu_{n+1}\right), d_{\infty}\left(\mu_{n+1}, \mu_{n+2}\right)\right. \\
& \left., d_{\infty}\left(\mu_{n+1}, \mu_{n+1}\right), \rho_{\infty}\left(\mu_{n}, F_{n+2}\left(\mu_{n+1}\right)\right)\right\} \\
\leqslant & \min \left\{d_{\infty}\left(\mu_{n}, \mu_{n+1}\right), d_{\infty}\left(\mu_{n}, \mu_{n+1}\right), d_{\infty}\left(\mu_{n+1}, \mu_{n+2}\right), 0, \rho_{\infty}\left(\mu_{n}, F_{n+2}\left(\mu_{n+1}\right)\right)\right\}=0 .
\end{aligned}
$$

Thus, from (2.12), we can get

$$
\mathrm{N}\left(\mu_{n}, \mu_{n+1}\right)=0 \text {. }
$$

By (2.10), (2.11), (2.13) and the nondecreasing character of $\phi$, we have

$$
d_{\infty}\left(\mu_{n+1}, \mu_{n+2}\right) \leqslant g\left(d_{\infty}\left(\mu_{n}, \mu_{n+1}\right), d_{\infty}\left(\mu_{n}, \mu_{n+1}\right), d_{\infty}\left(\mu_{n+1}, \mu_{n+2}\right), 0, d_{\infty}\left(\mu_{n}, \mu_{n+1}\right)+d_{\infty}\left(\mu_{n+1}, \mu_{n+2}\right)\right) .
$$


From Definition 2.1 (ii), we can conclude that

$$
d_{\infty}\left(\mu_{n+1}, \mu_{n+2}\right) \leqslant h d_{\infty}\left(\mu_{n}, \mu_{n+1}\right),
$$

where $0<\mathrm{h}<1$. By iteration, we have

$$
d_{\infty}\left(\mu_{n}, \mu_{n+1}\right) \leqslant h d\left(x_{n-1}, x_{n}\right) \leqslant \cdots \leqslant h^{n} d\left(\mu_{0}, \mu_{1}\right) .
$$

Furthermore, for $m>n$,

$$
\begin{aligned}
d\left(\mu_{n}, \mu_{m}\right) & \leqslant d_{\infty}\left(\mu_{n}, \mu_{n+1}\right)+d_{\infty}\left(\mu_{n+1}, \mu_{n+2}\right)+\cdots+d_{\infty}\left(\mu_{m-1}, \mu_{m}\right) \\
& \leqslant\left(h^{n}+h^{n-1}+\cdots+h^{m-1}\right) d_{\infty}\left(\mu_{0}, \mu_{1}\right) \leqslant \frac{h^{n}}{1-h} d_{\infty}\left(\mu_{0}, \mu_{1}\right) .
\end{aligned}
$$

It follows that $\left\{\mu_{n}\right\}$ is a Cauchy sequence in $\mathcal{C}(X)$. Since $X$ is compact, it implies that $X$ is complete. Thus there exists a $\mu_{*}$ such that $\lim _{n \rightarrow \infty} \mu_{n}=x_{*}$. Next, we show that $\left\{\mu_{*}\right\} \subset F_{i} \mu_{*}$ for all $i \in \mathbf{N}$.

Let $i \in \mathbf{N}$ be arbitrary. By (ii) and (iii) in Lemma 1.2, let us notice that

$$
\rho_{\infty}\left(\mu_{*}, F_{i}\left(\mu_{*}\right)\right) \leqslant d_{\infty}\left(\mu_{*}, \mu_{j}\right)+\rho_{\infty}\left(\mu_{j}, F_{i}\left(\mu_{*}\right)\right) \leqslant d_{\infty}\left(\mu_{*}, \mu_{j}\right)+d_{\infty}\left(F_{j}\left(\mu_{j-1}\right), F_{i}\left(\mu_{*}\right)\right),
$$

since $\mu_{j} \in F_{j}\left(\mu_{j-1}\right)$ for arbitrary natural numbers $j$ such that $i \neq j$. From

$$
\left|\rho_{\infty}\left(\mu_{*}, F_{i}\left(\mu_{*}\right)\right)-d_{\infty}\left(\mu_{*}, \mu_{j}\right)\right| \leqslant d_{\infty}\left(F_{j}\left(\mu_{j-1}\right), F_{i}\left(\mu_{*}\right)\right),
$$

(2.8), and the nondecreasing character of $\phi$, we have

$$
\phi\left(\left|\rho_{\infty}\left(\mu_{*}, F_{i}\left(\mu_{*}\right)\right)-d_{\infty}\left(\mu_{*}, \mu_{j}\right)\right|\right) \leqslant \phi\left(d_{\infty}\left(F_{j}\left(\mu_{j-1}\right), F_{i}\left(\mu_{*}\right)\right)\right) \leqslant \phi\left(M\left(\mu_{j-1}, \mu_{*}\right)\right)+L N\left(\mu_{j-1}, \mu_{*}\right),
$$

where

$$
\begin{aligned}
M\left(\mu_{j-1}, \mu_{*}\right)= & g\left(d_{\infty}\left(\mu_{j-1}, \mu_{*}\right), \rho_{\infty}\left(\mu_{j-1}, F_{j}\left(\mu_{j-1}\right)\right)\right. \\
& \left., \rho_{\infty}\left(\mu_{*}, F_{i}\left(\mu_{*}\right)\right), \rho_{\infty}\left(\mu_{*}, F_{j}\left(\mu_{j-1}\right)\right), \rho_{\infty}\left(\mu_{j-1}, F_{i}\left(\mu_{*}\right)\right)\right) \\
\leqslant & g\left(d_{\infty}\left(\mu_{j-1}, \mu_{*}\right), d_{\infty}\left(\mu_{j-1}, \mu_{j}\right), d_{\infty}\left(\mu_{*}, \mu_{j}\right)+\rho_{\infty}\left(\mu_{j}, F_{i}\left(\mu_{*}\right)\right)\right. \\
& \left.\left., d_{\infty}\left(\mu_{*}, \mu_{j}\right)+d_{\infty}\left(\mu_{j}, \mu_{j}\right)\right), d_{\infty}\left(\mu_{j-1}, \mu_{*}\right)+\rho_{\infty}\left(\mu_{*}, F_{i}\left(\mu_{*}\right)\right)\right)
\end{aligned}
$$

and

$$
\begin{aligned}
N\left(\mu_{j-1}, \mu_{*}\right)= & \min \left\{d_{\infty}\left(\mu_{j-1}, \mu_{*}\right), \rho_{\infty}\left(\mu_{j-1}, F_{j}\left(\mu_{j-1}\right)\right)\right. \\
& \left., \rho_{\infty}\left(\mu_{*}, F_{i}\left(\mu_{*}\right)\right), \rho_{\infty}\left(\mu_{*}, F_{j}\left(\mu_{j-1}\right)\right), \rho_{\infty}\left(\mu_{j-1}, F_{i}\left(\mu_{*}\right)\right)\right\} \\
\leqslant & \min \left\{d_{\infty}\left(\mu_{j-1}, \mu_{*}\right), d_{\infty}\left(\mu_{j-1}, \mu_{j}\right)\right), d_{\infty}\left(\mu_{*}, \mu_{j}\right)+\rho_{\infty}\left(\mu_{j}, F_{i}\left(\mu_{*}\right)\right) \\
& \left.\left., d_{\infty}\left(\mu_{*}, \mu_{j}\right)+d_{\infty}\left(\mu_{j}, \mu_{j}\right)\right), d_{\infty}\left(\mu_{j-1}, \mu_{*}\right)+\rho_{\infty}\left(\mu_{*}, F_{i}\left(\mu_{*}\right)\right)\right\} .
\end{aligned}
$$

Letting $j \rightarrow \infty$ in inequalities (2.14)-(2.16), we obtain

$$
\phi\left(\rho_{\infty}\left(\mu_{*}, F_{i}\left(\mu_{*}\right)\right)\right) \leqslant \phi\left(g\left(0,0, \rho_{\infty}\left(\mu_{*}, F_{i}\left(\mu_{*}\right)\right), 0, \rho_{\infty}\left(\mu_{*}, F_{i}\left(\mu_{*}\right)\right)\right)\right) .
$$

By the nondecreasing character of $\phi$, we have

$$
\rho_{\infty}\left(\mu_{*}, F_{i}\left(\mu_{*}\right)\right)<g\left(0,0, \rho_{\infty}\left(\mu_{*}, F_{i}\left(\mu_{*}\right)\right), 0, \rho_{\infty}\left(\mu_{*}, F_{i}\left(\mu_{*}\right)\right)\right) .
$$

Using (ii) in Definition 2.1, we can get $\rho_{\infty}\left(\mu_{*}, F_{i} \mu_{*}\right)=0$. Therefore, we have $\left\{\mu_{*}\right\} \subset F_{i} \mu_{*}$.

If in Theorem 2.4 we choose $\phi(t)=t$, we can get the following corollary. 
Corollary 2.5. Let $(\mathrm{X}, \mathrm{d})$ be a compact metric space and $\mathrm{g}$ be a $\mathrm{G}$-distance function and $\left\{\mathrm{F}_{i}\right\}_{i=1}^{\infty}$ be a sequence of self-mappings of $\mathcal{C}(X)$. Suppose that there exists an $\mathrm{L} \geqslant 0$ such that for each $\mu_{1}, \mu_{2} \in \mathcal{C}(X)$, and for arbitrary positive integers $i$ and $j, i \neq j$,

$$
d_{\infty}\left(F_{i}\left(\mu_{1}\right), F_{j}\left(\mu_{2}\right)\right) \leqslant M\left(\mu_{1}, \mu_{2}\right)+L N\left(\mu_{1}, \mu_{2}\right),
$$

where

$$
\begin{aligned}
M\left(\mu_{1}, \mu_{2}\right) & =g\left(d_{\infty}\left(\mu_{1}, \mu_{2}\right), \rho_{\infty}\left(\mu_{1}, F_{i}\left(\mu_{1}\right)\right), \rho_{\infty}\left(\mu_{2}, F_{j}\left(\mu_{2}\right)\right), \rho_{\infty}\left(\mu_{2}, F_{i}\left(\mu_{1}\right)\right), \rho_{\infty}\left(\mu_{1}, F_{j}\left(\mu_{2}\right)\right)\right), \\
N\left(\mu_{1}, \mu_{2}\right) & =\min \left\{d_{\infty}\left(\mu_{1}, \mu_{2}\right), \rho_{\infty}\left(\mu_{1}, F_{i}\left(\mu_{1}\right)\right), \rho_{\infty}\left(\mu_{2}, F_{j}\left(\mu_{2}\right)\right), \rho_{\infty}\left(\mu_{2}, F_{i}\left(\mu_{1}\right)\right), \rho_{\infty}\left(\mu_{1}, F_{j}\left(\mu_{2}\right)\right)\right\} .
\end{aligned}
$$

Then there exists a $\mu_{*} \in \mathcal{C}(X)$ such that $\mu_{*} \subseteq \mathrm{F}_{i}\left(\mu_{*}\right)$ for all $i \in N$.

Corollary 2.6. Let $(\mathrm{X}, \mathrm{d})$ be a compact metric space and $\mathrm{g}$ be $a \mathrm{G}$-distance function and $\left\{\mathrm{F}_{i}\right\}_{i=1}^{\infty}$ be a sequence of self-mappings of $\mathfrak{C}(\mathrm{X})$. Suppose that there exist $\phi \in \Psi$ and $\mathfrak{R}$-function $\varphi:[0, \infty) \rightarrow[0,1)$ such that for each $\mu_{1}, \mu_{2} \in \mathcal{C}(X)$, and for arbitrary positive integers $i$ and $j, i \neq j$,

$$
\phi\left(d_{\infty}\left(F_{i}\left(\mu_{1}\right), F_{j}\left(\mu_{2}\right)\right)\right) \leqslant \phi\left(M\left(\mu_{1}, \mu_{2}\right)\right)-\varphi\left(d_{\infty}\left(\mu_{1}, \mu_{2}\right)\right),
$$

where

$$
M\left(\mu_{1}, \mu_{2}\right)=g\left(d_{\infty}\left(\mu_{1}, \mu_{2}\right), \rho_{\infty}\left(\mu_{1}, F_{i}\left(\mu_{1}\right)\right), \rho_{\infty}\left(\mu_{2}, F_{j}\left(\mu_{2}\right)\right), \rho_{\infty}\left(\mu_{2}, F_{i}\left(\mu_{1}\right)\right), \rho_{\infty}\left(\mu_{1}, F_{j}\left(\mu_{2}\right)\right)\right) .
$$

Then there exists a $\mu_{*} \in \mathcal{C}(X)$ such that $\mu_{*} \subseteq \mathrm{F}_{\mathbf{i}}\left(\mu_{*}\right)$ for all $i \in N$.

Proof. Since

$$
\phi\left(d_{\infty}\left(F_{i}\left(\mu_{1}\right), F_{j}\left(\mu_{2}\right)\right)\right) \leqslant \phi\left(M\left(\mu_{1}, \mu_{2}\right)\right)-\varphi\left(d_{\infty}\left(\mu_{1}, \mu_{2}\right)\right) \leqslant \phi\left(M\left(\mu_{1}, \mu_{2}\right)\right)+L N\left(\mu_{1}, \mu_{2}\right),
$$

Hence, by using Theorem 2.4, there exists a point $\mu_{*}$ in $\mathcal{C}(X)$ such that $\left\{\mu_{*}\right\} \subset F_{i}\left(\mu_{*}\right)$.

If in Corollary 2.6 we chose $\phi(t)=t$, we can obtain the following corollary.

Corollary 2.7. Let $(\mathrm{X}, \mathrm{d})$ be a compact metric space and $\mathrm{g}$ be a $\mathrm{G}$-distance function and $\left\{\mathrm{F}_{i}\right\}_{i=1}^{\infty}$ be a sequence of self-mappings of $\mathfrak{C}(X)$. Suppose that there exists an $\mathfrak{R}$-function $\varphi:[0, \infty) \rightarrow[0,1)$ such that for each $\mu_{1}, \mu_{2} \in \mathcal{C}(X)$, and for arbitrary positive integers $i$ and $j, i \neq j$,

$$
d_{\infty}\left(F_{i}\left(\mu_{1}\right), F_{j}\left(\mu_{2}\right)\right) \leqslant M\left(\mu_{1}, \mu_{2}\right)-\varphi\left(d_{\infty}\left(\mu_{1}, \mu_{2}\right)\right),
$$

where

$$
M\left(\mu_{1}, \mu_{2}\right)=g\left(d_{\infty}\left(\mu_{1}, \mu_{2}\right), \rho_{\infty}\left(\mu_{1}, F_{i}\left(\mu_{1}\right)\right), \rho_{\infty}\left(\mu_{2}, F_{j}\left(\mu_{2}\right)\right), \rho_{\infty}\left(\mu_{2}, F_{i}\left(\mu_{1}\right)\right), \rho_{\infty}\left(\mu_{1}, F_{j}\left(\mu_{2}\right)\right)\right) .
$$

Then there exists a $\mu_{*} \in \mathcal{C}(X)$ such that $\mu_{*} \subseteq \mathrm{F}_{i}\left(\mu_{*}\right)$ for all $i \in N$.

\section{Fuzzy fixed point theorems under a $G^{\prime}$-distance function}

In this section, inspired by Constantin [17] and Chen et al. [11], we will show some fuzzy fixed point theorems on a space of fuzzy sets via a $G^{\prime}$-distance function. In what follows, we give the definition of $\mathrm{G}^{\prime}$-distance functions which are introduced by Chen et al. [11].

Definition 3.1. A function $g$ is said to be a $G^{\prime}$-distance function if $g:[0, \infty)^{5} \rightarrow[0, \infty)$ is continuous function with the following properties hold:

(i) $g$ is increasing in each co-ordinate variable;

(ii) $g(t, t, t, a t, b t) \leqslant t$ for every $t \in[0, \infty)$, where $a+b=2$. 
Now, we establish and prove the following fixed point theorem.

Theorem 3.2. Let $(\mathrm{X}, \mathrm{d})$ be a compact metric space and $\mathrm{g}$ be a $\mathrm{G}^{\prime}$-distance function and $\left\{\mathrm{F}_{\mathrm{i}}\right\}_{\mathrm{i}=1}^{\infty}$ be a sequence of self-mappings of $\mathfrak{C}(X)$. Suppose that there exist $\psi \in \Omega, \eta \in \Upsilon$ and $L \geqslant 0$ such that for each $\mu_{1}, \mu_{2} \in \mathcal{C}(X)$, and for arbitrary positive integers $i$ and $j, i \neq j$,

$$
d_{\infty}\left(F_{i}\left(\mu_{1}\right), F_{j}\left(\mu_{2}\right)\right) \leqslant \psi\left(M\left(\mu_{1}, \mu_{2}\right)\right)+\operatorname{L\eta }\left(N\left(\mu_{1}, \mu_{2}\right)\right),
$$

where

$$
\begin{aligned}
M\left(\mu_{1}, \mu_{2}\right) & =g\left(d_{\infty}\left(\mu_{1}, \mu_{2}\right), \rho_{\infty}\left(\mu_{1}, F_{i}\left(\mu_{1}\right)\right), \rho_{\infty}\left(\mu_{2}, F_{j}\left(\mu_{2}\right)\right), \rho_{\infty}\left(\mu_{2}, F_{i}\left(\mu_{1}\right)\right), \rho_{\infty}\left(\mu_{1}, F_{j}\left(\mu_{2}\right)\right)\right), \\
N\left(\mu_{1}, \mu_{2}\right) & =\min \left\{d_{\infty}\left(\mu_{1}, \mu_{2}\right), \rho_{\infty}\left(\mu_{1}, F_{i}\left(\mu_{1}\right)\right), \rho_{\infty}\left(\mu_{2}, F_{j}\left(\mu_{2}\right)\right), \rho_{\infty}\left(\mu_{2}, F_{i}\left(\mu_{1}\right)\right), \rho_{\infty}\left(\mu_{1}, F_{j}\left(\mu_{2}\right)\right)\right\} .
\end{aligned}
$$

Then there exists a $\mu_{*} \in \mathcal{C}(X)$ such that $\mu_{*} \subseteq \mathrm{F}_{\mathfrak{i}}\left(\mu_{*}\right)$ for all $i \in N$.

Proof. Let $\mu_{0} \in \mathcal{C}(X)$ and $\mu_{1} \subseteq F_{1}\left(\mu_{0}\right)$, by Lemma 1.4, there exists a $\mu_{2}$ such that $\mu_{2} \subseteq F_{2}\left(\mu_{1}\right)$ and

$$
d_{\infty}\left(\mu_{1}, \mu_{2}\right) \leqslant d_{\infty}\left(F_{1}\left(\mu_{0}\right), F_{2}\left(\mu_{1}\right)\right) .
$$

Again by Lemma 1.4, we can find $\mu_{3} \in \mathcal{C}(X)$ such that $\mu_{3} \subseteq F_{3}\left(\mu_{2}\right)$,

$$
d_{\infty}\left(\mu_{2}, \mu_{3}\right) \leqslant d_{\infty}\left(F_{2}\left(\mu_{1}\right), F_{3}\left(\mu_{2}\right)\right) .
$$

By induction, we produce a sequence $\left\{\mu_{n}\right\}$ of points of $\mathcal{C}(X)$ such that

$$
\left\{\begin{array}{l}
\mu_{n+2} \subseteq F_{n+2}\left(\mu_{n+1}\right), \\
d_{\infty}\left(\mu_{n+1}, \mu_{n+2}\right) \leqslant d_{\infty}\left(F_{n+1}\left(\mu_{n}\right), F_{n+2}\left(\mu_{n+1}\right)\right) .
\end{array}\right.
$$

Next, we prove that $\left\{\mu_{n}\right\}$ is a Cauchy sequence in $\mathcal{C}(X)$. In fact, for arbitrary positive integer $n$, by the inequality (3.1), the formula (3.2) and the properties of $\psi$, we have

$$
d_{\infty}\left(\mu_{n+1}, \mu_{n+2}\right) \leqslant d_{\infty}\left(F_{n+1}\left(\mu_{n}\right), F_{n+2}\left(\mu_{n+1}\right)\right) \leqslant \psi\left(M\left(\mu_{n}, \mu_{n+1}\right)\right)+\operatorname{L\eta }\left(N\left(\mu_{n}, \mu_{n+1}\right)\right),
$$

where

$$
\begin{aligned}
& M\left(\mu_{n}, \mu_{n+1}\right)= g\left(d_{\infty}\left(\mu_{n}, \mu_{n+1}\right), \rho_{\infty}\left(\mu_{n}, F_{n+1}\left(\mu_{n}\right)\right)\right. \\
&\left., \rho_{\infty}\left(\mu_{n+1}, F_{n+2}\left(\mu_{n+1}\right)\right), \rho_{\infty}\left(\mu_{n+1}, F_{n+1}\left(\mu_{n}\right)\right), \rho_{\infty}\left(\mu_{n}, F_{n+2}\left(\mu_{n+1}\right)\right)\right) \\
& \leqslant g\left(d_{\infty}\left(\mu_{n}, \mu_{n+1}\right), d_{\infty}\left(\mu_{n}, \mu_{n+1}\right), d_{\infty}\left(\mu_{n+1}, \mu_{n+2}\right)\right. \\
&\left., d_{\infty}\left(\mu_{n+1}, \mu_{n+1}\right), \rho_{\infty}\left(\mu_{n}, F_{n+2}\left(\mu_{n+1}\right)\right)\right) \\
& \leqslant g\left(d_{\infty}\left(\mu_{n}, \mu_{n+1}\right), d_{\infty}\left(\mu_{n}, \mu_{n+1}\right), d_{\infty}\left(\mu_{n+1}, \mu_{n+2}\right), 0, \rho_{\infty}\left(\mu_{n}, F_{n+2}\left(\mu_{n+1}\right)\right)\right) \\
& \leqslant g\left(d_{\infty}\left(\mu_{n}, \mu_{n+1}\right), d_{\infty}\left(\mu_{n}, \mu_{n+1}\right), d_{\infty}\left(\mu_{n+1}, \mu_{n+2}\right), 0\right. \\
&\left., d_{\infty}\left(\mu_{n}, \mu_{n+1}\right)+\rho_{\infty}\left(\mu_{n+1}, F_{n+2}\left(\mu_{n+1}\right)\right)\right) \\
& \leqslant g\left(d_{\infty}\left(\mu_{n}, \mu_{n+1}\right), d_{\infty}\left(\mu_{n}, \mu_{n+1}\right), d_{\infty}\left(\mu_{n+1}, \mu_{n+2}\right), 0\right. \\
&\left., d_{\infty}\left(\mu_{n}, \mu_{n+1}\right)+d_{\infty}\left(\mu_{n+1}, \mu_{n+2}\right)\right), \\
& N\left(\mu_{n}, \mu_{n+1}\right)=\min \left\{d_{\infty}\left(\mu_{n}, \mu_{n+1}\right), \rho_{\infty}\left(\mu_{n}, F_{n+1}\left(\mu_{n}\right)\right)\right. \\
&\left., \rho_{\infty}\left(\mu_{n+1}, F_{n+2}\left(\mu_{n+1}\right)\right), \rho_{\infty}\left(\mu_{n+1}, F_{n+1}\left(\mu_{n}\right)\right), \rho_{\infty}\left(\mu_{n}, F_{n+2}\left(\mu_{n+1}\right)\right)\right\} \\
& \leqslant \min \left\{d_{\infty}\left(\mu_{n}, \mu_{n+1}\right), d_{\infty}\left(\mu_{n}, \mu_{n+1}\right), d_{\infty}\left(\mu_{n+1}, \mu_{n+2}\right)\right. \\
&\left., d_{\infty}\left(\mu_{n+1}, \mu_{n+1}\right), \rho_{\infty}\left(\mu_{n}, F_{n+2}\left(\mu_{n+1}\right)\right)\right\} \\
& \leqslant \min \left\{d_{\infty}\left(\mu_{n}, \mu_{n+1}\right), d_{\infty}\left(\mu_{n}, \mu_{n+1}\right), d_{\infty}\left(\mu_{n+1}, \mu_{n+2}\right), 0, \rho_{\infty}\left(\mu_{n}, F_{n+2}\left(\mu_{n+1}\right)\right)\right\}=0 .
\end{aligned}
$$


Thus, from (3.5) and the properties of $\eta$, we can get

$$
\eta\left(N\left(\mu_{n}, \mu_{n+1}\right)\right)=0 .
$$

By (3.3), (3.4), (3.6), and the nondecreasing character of $\psi$, we have

$$
\begin{aligned}
d_{\infty}\left(\mu_{n+1}, \mu_{n+2}\right) \leqslant & \psi\left(g \left(d_{\infty}\left(\mu_{n}, \mu_{n+1}\right), d_{\infty}\left(\mu_{n}, \mu_{n+1}\right), d_{\infty}\left(\mu_{n+1}, \mu_{n+2}\right), 0\right.\right. \\
& \left.\left., d_{\infty}\left(\mu_{n}, \mu_{n+1}\right)+d_{\infty}\left(\mu_{n+1}, \mu_{n+2}\right)\right)\right) .
\end{aligned}
$$

Now, we prove that $d_{\infty}\left(\mu_{n+1}, \mu_{n+2}\right) \leqslant d_{\infty}\left(\mu_{n}, \mu_{n+1}\right)$. If $d_{\infty}\left(\mu_{n+1}, \mu_{n+2}\right)>d_{\infty}\left(\mu_{n}, \mu_{n+1}\right)$, then from (3.7) and the nondecreasing character of $\psi$ and $g$, we can get

$$
d_{\infty}\left(\mu_{n+1}, \mu_{n+2}\right) \leqslant \psi\left(g\left(d_{\infty}\left(\mu_{n+1}, \mu_{n+2}\right), d_{\infty}\left(\mu_{n+1}, \mu_{n+2}\right), d_{\infty}\left(\mu_{n+1}, \mu_{n+2}\right), 0,2 d_{\infty}\left(\mu_{n+1}, \mu_{n+2}\right)\right)\right) .
$$

Since $g$ is a $G^{\prime}$-distance function, by (ii) of Definition 3.1, we can conclude that

$$
\begin{aligned}
d_{\infty}\left(\mu_{n+1}, \mu_{n+2}\right) \leqslant & \psi\left(g \left(d_{\infty}\left(\mu_{n+1}, \mu_{n+2}\right), d_{\infty}\left(\mu_{n+1}, \mu_{n+2}\right), d_{\infty}\left(\mu_{n+1}, \mu_{n+2}\right), 0 \times d_{\infty}\left(\mu_{n+1}, \mu_{n+2}\right)\right.\right. \\
& \left.\left., 2 d_{\infty}\left(\mu_{n+1}, \mu_{n+2}\right)\right)\right) \leqslant \psi\left(d_{\infty}\left(\mu_{n+1}, \mu_{n+2}\right)\right)<d_{\infty}\left(\mu_{n+1}, \mu_{n+2}\right)
\end{aligned}
$$

which is a contradiction. Hence, we have $d_{\infty}\left(\mu_{n+1}, \mu_{n+2}\right) \leqslant d_{\infty}\left(\mu_{n}, \mu_{n+1}\right)$. By (3.8) and the nondecreasing character of $\psi$, we have $d_{\infty}\left(\mu_{n+1}, \mu_{n+2}\right) \leqslant \psi\left(d_{\infty}\left(\mu_{n+1}, \mu_{n+2}\right)\right) \leqslant \psi\left(d_{\infty}\left(\mu_{n}, \mu_{n+1}\right)\right)$. Therefore, for all $n$, we can conclude that $d_{\infty}\left(\mu_{n}, \mu_{n+1}\right) \leqslant d_{\infty}\left(\mu_{n-1}, \mu_{n}\right)$. Therefore, for positive integers $m, n(n>m)$, we get

$$
\begin{aligned}
d_{\infty}\left(\mu_{m}, \mu_{n}\right) & \leqslant d_{\infty}\left(\mu_{m}, \mu_{m+1}\right)+\cdots+d_{\infty}\left(\mu_{n-1}, \mu_{n}\right) \\
& <\psi^{m}\left(d_{\infty}\left(\mu_{0}, \mu_{1}\right)\right)+\cdots+\psi^{n-1}\left(d_{\infty}\left(\mu_{0}, \mu_{1}\right)\right) \\
& =\sum_{k=m}^{n-1} \psi^{n}\left(d_{\infty}\left(\mu_{0}, \mu_{1}\right)\right) .
\end{aligned}
$$

In (3.9), as $m, n \rightarrow \infty$, we have $d_{\infty}\left(\mu_{m}, \mu_{n}\right) \rightarrow 0$. It follows that $\left\{\mu_{n}\right\}$ is a Cauchy sequence in $\mathcal{C}(X)$. Since $X$ is compact, it implies that $X$ is complete. Thus there exists a $\mu_{*}$ such that $\lim _{n \rightarrow \infty} \mu_{n}=x_{*}$. Next, we show that $\left\{\mu_{*}\right\} \subset F_{i} \mu_{*}$ for all $i \in \mathbf{N}$.

Let $i \in \mathbf{N}$ be arbitrary. By (ii) and (iii) in Lemma 1.2, let us notice that

$$
\begin{aligned}
\rho_{\infty}\left(\mu_{*}, F_{i}\left(\mu_{*}\right)\right) & \leqslant d_{\infty}\left(\mu_{*}, \mu_{j}\right)+\rho_{\infty}\left(\mu_{j}, F_{i}\left(\mu_{*}\right)\right) \\
& \leqslant d_{\infty}\left(\mu_{*}, \mu_{j}\right)+d_{\infty}\left(F_{j}\left(\mu_{j-1}\right), F_{i}\left(\mu_{*}\right)\right) \\
& \leqslant d_{\infty}\left(\mu_{*}, \mu_{j}\right)+\psi\left(M\left(\mu_{j-1}, \mu_{*}\right)\right)+\operatorname{L\eta }\left(N\left(\mu_{j-1}, \mu_{*}\right)\right),
\end{aligned}
$$

since $\mu_{j} \in F_{j}\left(\mu_{j-1}\right)$ for arbitrary natural numbers $j$ such that $i \neq j$, where

$$
\begin{aligned}
M\left(\mu_{j-1}, \mu_{*}\right)= & g\left(d_{\infty}\left(\mu_{j-1}, \mu_{*}\right), \rho_{\infty}\left(\mu_{j-1}, F_{j}\left(\mu_{j-1}\right)\right),\right. \\
& \left., \rho_{\infty}\left(\mu_{*}, F_{i}\left(\mu_{*}\right)\right), \rho_{\infty}\left(\mu_{*}, F_{j}\left(\mu_{j-1}\right)\right), \rho_{\infty}\left(\mu_{j-1}, F_{i}\left(\mu_{*}\right)\right)\right) \\
\leqslant & g\left(d_{\infty}\left(\mu_{j-1}, \mu_{*}\right), d_{\infty}\left(\mu_{j-1}, \mu_{j}\right), d_{\infty}\left(\mu_{*}, \mu_{j}\right)+\rho_{\infty}\left(\mu_{j}, F_{i}\left(\mu_{*}\right)\right)\right. \\
& \left., d_{\infty}\left(\mu_{*}, \mu_{j}\right)+d_{\infty}\left(\mu_{j}, \mu_{j}\right), d_{\infty}\left(\mu_{j-1}, \mu_{*}\right)+\rho_{\infty}\left(\mu_{*}, F_{i}\left(\mu_{*}\right)\right)\right)
\end{aligned}
$$

and

$$
\begin{aligned}
N\left(\mu_{j-1}, \mu_{*}\right)= & \min \left\{d_{\infty}\left(\mu_{j-1}, \mu_{*}\right), \rho_{\infty}\left(\mu_{j-1}, F_{j}\left(\mu_{j-1}\right)\right)\right. \\
& \left., \rho_{\infty}\left(\mu_{*}, F_{i}\left(\mu_{*}\right)\right), \rho_{\infty}\left(\mu_{*}, F_{j}\left(\mu_{j-1}\right)\right), \rho_{\infty}\left(\mu_{j-1}, F_{i}\left(\mu_{*}\right)\right)\right\} \\
\leqslant & \min \left\{d_{\infty}\left(\mu_{j-1}, \mu_{*}\right), d_{\infty}\left(\mu_{j-1}, \mu_{j}\right), d_{\infty}\left(\mu_{*}, \mu_{j}\right)+\rho_{\infty}\left(\mu_{j}, F_{i}\left(\mu_{*}\right)\right)\right. \\
& \left., d_{\infty}\left(\mu_{*}, \mu_{j}\right)+d_{\infty}\left(\mu_{j}, \mu_{j}\right), d_{\infty}\left(\mu_{j-1}, \mu_{*}\right)+\rho_{\infty}\left(\mu_{*}, F_{i}\left(\mu_{*}\right)\right)\right\}
\end{aligned}
$$


Letting $j \rightarrow \infty$ in inequalities (3.10)-(3.12), we obtain

$$
\rho_{\infty}\left(\mu_{*}, F_{i}\left(\mu_{*}\right)\right) \leqslant 0+\psi\left(g\left(0,0, \rho_{\infty}\left(\mu_{*}, F_{i}\left(\mu_{*}\right)\right), 0, \rho_{\infty}\left(\mu_{*}, F_{i}\left(\mu_{*}\right)\right)\right)\right)+\operatorname{L\eta }(0) .
$$

By the nondecreasing character of $\psi$, we have

$$
\begin{aligned}
\rho_{\infty}\left(\mu_{*}, F_{i}\left(\mu_{*}\right)\right) & \leqslant g\left(0,0, \rho_{\infty}\left(\mu_{*}, F_{i}\left(\mu_{*}\right)\right), 0, \rho_{\infty}\left(\mu_{*}, F_{i}\left(\mu_{*}\right)\right)\right) \\
& \leqslant \psi\left(g\left(\rho_{\infty}\left(\mu_{*}, F_{i}\left(\mu_{*}\right)\right), \rho_{\infty}\left(\mu_{*}, F_{i}\left(\mu_{*}\right)\right), \rho_{\infty}\left(\mu_{*}, F_{i}\left(\mu_{*}\right)\right), \rho_{\infty}\left(\mu_{*}, F_{i}\left(\mu_{*}\right)\right), \rho_{\infty}\left(\mu_{*}, F_{i}\left(\mu_{*}\right)\right)\right)\right) \\
& \leqslant \psi\left(\rho_{\infty}\left(\mu_{*}, F_{i}\left(\mu_{*}\right)\right)\right)<\rho_{\infty}\left(\mu_{*}, F_{i}\left(\mu_{*}\right)\right)
\end{aligned}
$$

which is a contradiction. Hence, we can get $\rho_{\infty}\left(\mu_{*}, F_{i} \mu_{*}\right)=0$. Therefore, we have $\left\{\mu_{*}\right\} \subset F_{i} \mu_{*}$.

If in Theorem 3.2 we choose $L=0$, then we can get the following corollary.

Corollary 3.3. Let $(\mathrm{X}, \mathrm{d})$ be a compact metric space and $\mathrm{g}$ be $a \mathrm{G}^{\prime}$-distance function and $\left\{\mathrm{F}_{\mathrm{i}}\right\}_{i=1}^{\infty}$ be a sequence of self-mappings of $\mathcal{C}(X)$. Suppose that there exists $a \psi \in \Omega$ such that for each $\mu_{1}, \mu_{2} \in \mathcal{C}(X)$, and for arbitrary positive integers $i$ and $j, i \neq j$,

$$
d_{\infty}\left(F_{i}\left(\mu_{1}\right), F_{j}\left(\mu_{2}\right)\right) \leqslant \psi\left(M\left(\mu_{1}, \mu_{2}\right)\right),
$$

where

$$
M\left(\mu_{1}, \mu_{2}\right)=g\left(d_{\infty}\left(\mu_{1}, \mu_{2}\right), \rho_{\infty}\left(\mu_{1}, F_{i}\left(\mu_{1}\right)\right), \rho_{\infty}\left(\mu_{2}, F_{j}\left(\mu_{2}\right)\right), \rho_{\infty}\left(\mu_{2}, F_{i}\left(\mu_{1}\right)\right), \rho_{\infty}\left(\mu_{1}, F_{j}\left(\mu_{2}\right)\right)\right) .
$$

Then there exists a $\mu_{*} \in \mathcal{C}(X)$ such that $\mu_{*} \subseteq \mathrm{F}_{\mathfrak{i}}\left(\mu_{*}\right)$ for all $\mathrm{i} \in N$.

Remark 3.4. If in Corollary 3.3, we choose $g\left(x_{1}, x_{2}, x_{3}, x_{4}, x_{5}\right)=\max \left\{x_{1}, x_{2}, x_{3}, \frac{x_{4}+x_{5}}{2}\right\}$, then we can get Theorem 1.8.

If in Corollary 3.3, we choose $\psi(t)=q t$ where $0<q<1$, then we can get the following corollary.

Corollary 3.5. Let $(\mathrm{X}, \mathrm{d})$ be a compact metric space and $\mathrm{g}$ be $a \mathrm{G}^{\prime}$-distance function and $\left\{\mathrm{F}_{\mathrm{i}}\right\}_{i=1}^{\infty}$ be a sequence of self-mappings of $\mathrm{C}(\mathrm{X})$. Suppose that there exists a $\mathrm{q} \in(0,1)$ such that for each $\mu_{1}, \mu_{2} \in \mathfrak{C}(\mathrm{X})$, and for arbitrary positive integers $i$ and $j, i \neq j$,

$$
d_{\infty}\left(F_{i}\left(\mu_{1}\right), F_{j}\left(\mu_{2}\right)\right) \leqslant q\left(M\left(\mu_{1}, \mu_{2}\right)\right),
$$

where

$$
M\left(\mu_{1}, \mu_{2}\right)=g\left(d_{\infty}\left(\mu_{1}, \mu_{2}\right), \rho_{\infty}\left(\mu_{1}, F_{i}\left(\mu_{1}\right)\right), \rho_{\infty}\left(\mu_{2}, F_{j}\left(\mu_{2}\right)\right), \rho_{\infty}\left(\mu_{2}, F_{i}\left(\mu_{1}\right)\right), \rho_{\infty}\left(\mu_{1}, F_{j}\left(\mu_{2}\right)\right)\right) .
$$

Then there exists a $\mu_{*} \in \mathcal{C}(X)$ such that $\mu_{*} \subseteq \mathrm{F}_{\boldsymbol{i}}\left(\mu_{*}\right)$ for all $i \in N$.

If in Corollary 3.5, we choose $g\left(x_{1}, x_{2}, x_{3}, x_{4}, x_{5}\right)=\max \left\{x_{1}, x_{2}, x_{3}, \frac{x_{4}+x_{5}}{2}\right\}$, then we can get the following corollary.

Corollary 3.6. Let $(\mathrm{X}, \mathrm{d})$ be a compact metric space and $\mathrm{g}$ be a $\mathrm{G}^{\prime}$-distance function and $\left\{\mathrm{F}_{\mathrm{i}}\right\}_{i=1}^{\infty}$ be a sequence of self-mappings of $\mathrm{C}(\mathrm{X})$. Suppose that there exists a $\mathrm{q} \in(0,1)$ such that for each $\mu_{1}, \mu_{2} \in \mathfrak{C}(\mathrm{X})$, and for arbitrary positive integers $i$ and $j, i \neq j$,

$$
d_{\infty}\left(F_{i}\left(\mu_{1}\right), F_{j}\left(\mu_{2}\right)\right) \leqslant q\left(\max \left\{d_{\infty}\left(\mu_{1}, \mu_{2}\right), \rho_{\infty}\left(\mu_{1}, F_{i}\left(\mu_{1}\right)\right), \rho_{\infty}\left(\mu_{2}, F_{j}\left(\mu_{2}\right)\right), \frac{\rho_{\infty}\left(\mu_{2}, F_{i}\left(\mu_{1}\right)\right)+\rho_{\infty}\left(\mu_{1}, F_{j}\left(\mu_{2}\right)\right)}{2}\right\}\right) .
$$

Then there exists a $\mu_{*} \in \mathcal{C}(X)$ such that $\mu_{*} \subseteq \mathrm{F}_{i}\left(\mu_{*}\right)$ for all $i \in N$.

\section{Applications}

In this section, we mainly want to give some applications by using our mainly results. Firstly, we give an application to illustrate the usefulness of Theorem 2.2. 
Theorem 4.1. Let $(\mathrm{X}, \mathrm{d})$ be a compact metric space and $\left\{\mathrm{F}_{\mathrm{i}}\right\}_{i=1}^{\infty}$ a sequence of self-mappings of $\mathrm{C}(\mathrm{X})$ satisfying the following conditions:

$$
\begin{aligned}
{\left[d_{\infty}\left(F_{i}\left(\mu_{1}\right), F_{j}\left(\mu_{2}\right)\right)\right]^{2} \leqslant } & \alpha_{1}\left[d_{\infty}\left(\mu_{1}, \mu_{2}\right)\right]^{2}+\alpha_{2} \rho\left(\mu_{1}, F_{i}\left(\mu_{1}\right)\right) \rho\left(\mu_{2}, F_{j}\left(\mu_{2}\right)\right)+\alpha_{3} \rho\left(\mu_{2}, F_{i}\left(\mu_{1}\right)\right) \rho\left(\mu_{1}, F_{j}\left(\mu_{2}\right)\right) \\
& +\alpha_{4} d_{\infty}\left(\mu_{1}, \mu_{2}\right) \rho\left(\mu_{1}, F_{i}\left(\mu_{1}\right)\right)+\alpha_{5} d_{\infty}\left(\mu_{1}, \mu_{2}\right) \rho\left(\mu_{2}, F_{j}\left(\mu_{2}\right)\right),
\end{aligned}
$$

where $\alpha_{i}>0(i=1,2,3,4,5), \alpha_{1}+\alpha_{2}+\alpha_{4}+\alpha_{5}<1, \alpha_{1}+\alpha_{3}<1$. Then there exists a point $\mu_{*}$ in $\mathcal{C}(X)$ such that $\left\{\mu_{*}\right\} \subset \mathrm{F}_{i}\left(\mu_{*}\right)$.

Proof. We can consider the function $\mathrm{g}:[0, \infty)^{5} \rightarrow[0, \infty)$ defined by

$$
g\left(x_{1}, x_{2}, x_{3}, x_{4}, x_{5}\right)=\left[\alpha_{1} x_{1}^{2}+\alpha_{2} x_{2} x_{3}+\alpha_{3} x_{4} x_{5}+\alpha_{4} x_{1} x_{2}+\alpha_{5} x_{1} x_{3}\right]^{\frac{1}{2}} .
$$

Next, we prove $g$ is a G-distance function. Firstly, obviously, $g$ is nondecreasing in the $2^{\text {nd }}, 3^{\text {rd }}, 4^{\text {th }}$, and $5^{\text {th }}$ variables. Secondly,

$$
u \leqslant g(v, u, v, 0, u+v)=\left[\alpha_{1} v^{2}+\alpha_{2} u v+\alpha_{4} u v+\alpha_{5} v^{2}\right]^{\frac{1}{2}}=\left[\left(\alpha_{1}+\alpha_{5}\right) v^{2}+\left(\alpha_{2}+\alpha_{4}\right) u v\right]^{\frac{1}{2}} .
$$

If $u \leqslant v$, from $(4.1)$, we can get

$$
u^{2} \leqslant\left(\alpha_{1}+\alpha_{5}\right) v^{2}+\left(\alpha_{2}+\alpha_{4}\right) u v \leqslant\left(\alpha_{1}+\alpha_{2}+\alpha_{4}+\alpha_{5}\right) v^{2}
$$

Hence, there exists $h=\sqrt{\alpha_{1}+\alpha_{2}+\alpha_{4}+\alpha_{5}}<1$ such that $u \leqslant h v$, where $0<h<1$. If $u>v$, from (4.1), we can get

$$
u^{2} \leqslant\left(\alpha_{1}+\alpha_{5}\right) v^{2}+\left(\alpha_{2}+\alpha_{4}\right) u v<\left(\alpha_{1}+\alpha_{2}+\alpha_{4}+\alpha_{5}\right) u^{2}<u^{2},
$$

which is a contradiction. Therefore, (ii) of Definition 2.1 holds. Thirdly, since $u \leqslant g(u, 0,0, u, u)=$ $\left[\left(\alpha_{1}+\alpha_{3}\right) u^{2}\right]^{\frac{1}{2}}=\sqrt{\alpha_{1}+\alpha_{3}} u<u$, which is a contradiction. Hence, $u=0$. If in Theorem 2.2 we choose $\mathrm{L}=0$ and $\phi(\mathrm{t})=\mathrm{t}$, Theorem 4.1 is satisfied with all conditions of Theorem 2.2. Hence, there exists a point $\mu_{*}$ in $\mathcal{C}(X)$ such that $\left\{\mu_{*}\right\} \subset F_{i} \mu^{*}$.

Secondly, we give an application to illustrate the usefulness of Theorem 3.2 by slightly modifying Theorem 4.1.

Theorem 4.2. Let $(\mathrm{X}, \mathrm{d})$ be a compact metric space and $\left\{\mathrm{F}_{i}\right\}_{i=1}^{\infty}$ is a sequence of self-mappings of $\mathcal{C}(\mathrm{X})$ satisfying the following conditions:

$$
\begin{aligned}
{\left[d_{\infty}\left(F_{i}\left(\mu_{1}\right), F_{j}\left(\mu_{2}\right)\right)\right]^{2} \leqslant } & q\left\{\alpha_{1}\left[d_{\infty}\left(\mu_{1}, \mu_{2}\right)\right]^{2}+\alpha_{2} \rho\left(\mu_{1}, F_{i}\left(\mu_{1}\right)\right) \rho\left(\mu_{2}, F_{j}\left(\mu_{2}\right)\right)\right. \\
& \left.+\alpha_{3} d_{\infty}\left(\mu_{1}, \mu_{2}\right) \rho\left(\mu_{1}, F_{i}\left(\mu_{1}\right)\right)+\alpha_{4} d_{\infty}\left(\mu_{1}, \mu_{2}\right) \rho\left(\mu_{2}, F_{j}\left(\mu_{2}\right)\right)\right\},
\end{aligned}
$$

where $\mathrm{q} \in(0,1), \alpha_{i}>0(i=1,2,3,4)$, and $\alpha_{1}+\alpha_{2}+\alpha_{3}+\alpha_{4} \leqslant 1$. Then there exists a point $\mu_{*}$ in $\mathcal{C}(X)$ such that $\left\{\mu_{*}\right\} \subset \mathrm{F}_{\mathfrak{i}}\left(\mu_{*}\right)$.

Proof. We can consider the function $\mathrm{g}:[0, \infty)^{5} \rightarrow[0, \infty)$ defined by

$$
g\left(x_{1}, x_{2}, x_{3}, x_{4}, x_{5}\right)=\left[\alpha_{1} x_{1}^{2}+\alpha_{2} x_{2} x_{3}+\alpha_{3} x_{1} x_{2}+\alpha_{4} x_{1} x_{3}\right]^{\frac{1}{2}} .
$$

Next, we prove $g$ is a $G^{\prime}$-distance function. Firstly, obviously, $g$ is nondecreasing in the each co-ordinate variable. Secondly,

$$
g(t, t, t, a t, b t)=\left[\alpha_{1} v^{2}+\alpha_{2} t^{2}+\alpha_{3} t^{2}+\alpha_{4} t^{2}\right]^{\frac{1}{2}}=\left[\left(\alpha_{1}+\alpha_{2}+\alpha_{3}+\alpha_{4}\right) t^{2}\right]^{\frac{1}{2}}=\left(\alpha_{1}+\alpha_{2}+\alpha_{3}+\alpha_{4}\right)^{\frac{1}{2}} t \leqslant t .
$$

If in Theorem 3.2, we choose $L=0$ and $\psi(t)=q t(0<q<1)$, Theorem 4.2 is satisfied with all conditions of Theorem 3.2. Hence, there exists a point $\mu_{*}$ in $\mathcal{C}(X)$ such that $\left\{\mu_{*}\right\} \subset F_{i} \mu^{*}$. 


\section{Acknowledgment}

The authors thank the editor and the referees for their valuable comments and suggestions. This work was supported by National Natural Science Foundation of China (Grant No. 11461043, 11571370 and 11601525), and supported partly by the Provincial Natural Science Foundation of Jiangxi, China (20161BAB201009) and the Science and Technology Project of Educational Commission of Jiangxi Province, China (150013) and Hunan Provincial Innovation Foundation For Postgraduate (Grant No. CX2016B037) and Specialized Research Fund for the Doctoral Program of Higher Education of China (Grant No. 20120162110021).

\section{References}

[1] M. Abbas, D. Turkoglu, Fixed point theorem for a generalized contractive fuzzy mapping, J. Intell. Fuzzy Systems, 26 (2014), 33-36. 1

[2] A. Azam, M. Arshad, A note on "Fixed point theorems for fuzzy mappings" by P. Vijayaraju and M. Marudai [MR1979608 (2004d:26033)], Fuzzy Sets and Systems, 161 (2010), 1145-1149.

[3] A. Azam, M. Arshad, I. Beg, Fixed points of fuzzy contractive and fuzzy locally contractive maps, Chaos Solitons Fractals, 42 (2009), 2836-2841.

[4] A. Azam, M. Arshad, P. Vetro, On a pair of fuzzy ф-contractive mappings, Math. Comput. Modelling, 52 (2010), 207-214.

[5] A. Azam, I. Beg, Common fixed points of fuzzy maps, Math. Comput. Modelling, 49 (2009), 1331-1336.

[6] A. Azam, M. Rashid, Fuzzy fixed points of contractive fuzzy mappings, J. Comput. Anal. Appl., 15 (2013), $1006-1014$.

[7] A. Azam, M. Rashid, A fuzzy coincidence theorem with applications in a function space., J. Intell. Fuzzy Systems, 27 (2014), 1775-1781.

[8] M. Arshad, A. Shoaib, Fixed points of multivalued mappings in fuzzy metric spaces, Proceedings of the World Congress on Engineering, WCE, London, U.K., 1 (2012), 4-6.

[9] A. Azam, M. Waseem, M. Rashid, Fixed point theorems for fuzzy contractive mappings in quasi-pseudo-metric spaces, Fixed Point Theory Appl., 2014 (2014), 14 pages. 1

[10] J.-H. Chen, X.-J. Huang, Coupled fixed point theorems for $(\alpha, \varphi) \mathrm{g}$-contractive type mappings in partially ordered G-metric spaces, Open Math., 13 (2015), 877-888. 1

[11] J.-H. Chen, X.-J. Huang, Fixed point theorems for fuzzy mappings in metric spaces with an application, J. Inequal. Appl., 2015 (2015), 21 pages. 1, 2, 3

[12] J.-H. Chen, X.-J. Huang, S.-J. Li, Fixed point theorems for cyclic contractive mappings via altering distance functions in metric-like spaces, Open Math., 14 (2016), 857-874. 1

[13] J.-H. Chen, X.-H. Tang, Generalizations of Darbo's fixed point theorem via simulation functions with application to functional integral equations, J. Comput. Appl. Math., 296 (2016), 564-575. 1

[14] J.-H. Chen, X.-H. Tang, Z. Gao, Existence of multiple solutions for modified Schrödinger-Kirchhoff-Poisson type systems via perturbation method with sign-changing potential, Comput. Math. Appl., 73 (2017), 505-519. 1

[15] B. T. Cheng, X.-H. Tang, High energy solutions of modified quasilinear fourth-order elliptic equations with sign-changing potential, Comput. Math. Appl., 73 (2017), 27-36. 1

[16] L. Ćirić, M. Abbas, B. Damjanović, R. Saadati, Common fuzzy fixed point theorems in ordered metric spaces, Math. Comput. Modelling, 53 (2011), 1737-1741. 1

[17] A. Constantin, Common fixed points of weakly commuting mappings in 2-metric spaces, Math. Japon., 36 (1991), $507-$ 514. 1, 2, 3

[18] P. Diamond, P. Kloeden, Metric spaces of fuzzy sets, Theory and applications, World Scientific Publishing Co., Inc., River Edge, NJ, (1994). 1

[19] W.-S. Du, On coincidence point and fixed point theorems for nonlinear multivalued maps, Topology Appl., 159 (2012), 49-56. 1

[20] V. D. Estruch, A. Vidal, A note on fixed fuzzy points for fuzzy mappings, Proceedings of the "II Italian-Spanish Congress on General Topology and its Applications" (Italian), Trieste, (1999),Rend. Istit. Mat. Univ. Trieste, 32 (2001), 39-45. 1

[21] S. Heilpern, Fuzzy mappings and fixed point theorem, J. Math. Anal. Appl., 83 (1981), 566-569. 1

[22] B. S. Lee, S. J. Cho, A fixed point theorem for contractive-type fuzzy mappings, Fuzzy Sets and Systems, 61 (1994), 309-312. 1

[23] H.-L. Liu, H.-B. Chen, X.-X. Yang, Multiple solutions for superlinear Schrödinger-Poisson system with sign-changing potential and nonlinearity, Comput. Math. Appl., 68 (2014), 1982-1990. 1

[24] S. B. Nadler, Jr., Multi-valued contraction mappings, Pacific J. Math., 30 (1969), 475-488. 1

[25] J. Y. Park, J. U. Jeong, Common fixed points of fuzzy mappings, Fuzzy Sets and Systems, 59 (1993), 231-235.

[26] J. Y. Park, J. U. Jeong, Fixed point theorems for fuzzy mappings, Fuzzy Sets and Systems, 87 (1997), 111-116. 1 
[27] D. Qiu, L. Shu, Supremum metric on the space of fuzzy sets and common fixed point theorems for fuzzy mappings, Inform. Sci., 178 (2008), 3595-3604. 1, 1, 1.2, 1.3, 1

[28] D. Qiu, L. Shu, J. Guan, Common fixed point theorems for fuzzy mappings under $\Phi$-contraction condition, Chaos Solitons Fractals, 41 (2009), 360-367. 1, 1, 1.2, 1.4, 1

[29] R. A. Rashwan, M. A. Ahmad, Common fixed point theorems for fuzzy mappings, Arch. Math. (Brno), 38 (2002), 219-226. 1

[30] S. Sedghi, N. Shobe, I. Altun, A fixed fuzzy point for fuzzy mappings in complete metric spaces, Math. Commun., 13 (2008), 289-294. 1

[31] S. Suantai, N. Petrot, W. Saksirikun, Fuzzy fixed point theorems on the complete fuzzy spaces under supremum metric, Fixed Point Theory Appl., 2015 (2015), 13 pages. 1, 1, 1

[32] X. H. Tang, Infinitely many solutions for semilinear Schrödinger equations with sign-changing potential and nonlinearity, J. Math. Anal. Appl., 401 (2013), 407-415. 1

[33] X. H. Tang, Non-Nehari manifold method for superlinear Schrödinger equation, Taiwanese J. Math., 18 (2014), 1957-1979. 1

[34] L. A. Zadeh, Fuzzy sets, Information and Control, 8 (1965),338-353. 1

[35] J. Zhang, X.-H. Tang, W. Zhang, Existence of multiple solutions of Kirchhoff type equation with sign-changing potential, Appl. Math. Comput., 242 (2014), 491-499. 1

[36] J. Zhang, X.-H. Tang, W. Zhang, Infinitely many solutions of quasilinear Schrödinger equation with sign-changing potential, J. Math. Anal. Appl., 420 (2014), 1762-1775.

[37] W. Zhang, X.-H. Tang, J. Zhang, Ground state solutions for a diffusion system, Comput. Math. Appl., 69 (2015), 337-346. 1 